\title{
Frontières
}

\section{La mort de Lady Diana}

Un exemple de deuil public

\section{Joseph J. Lévy et Isabelle Toupin}

Volume 16, numéro 2, printemps 2004

Deuil, blessure vive

URI : https://id.erudit.org/iderudit/1074113ar

DOI : https://doi.org/10.7202/1074113ar

Aller au sommaire du numéro

Éditeur(s)

Université du Québec à Montréal

ISSN

1180-3479 (imprimé)

1916-0976 (numérique)

Découvrir la revue

Citer cet article

Lévy, J. J. \& Toupin, I. (2004). La mort de Lady Diana : un exemple de deuil public. Frontières, 16(2), 33-37. https://doi.org/10.7202/1074113ar
Résumé de l'article

L'expression du deuil public s'est manifestée de façon particulièrement éloquente lors de la mort de la princesse Diana en 1997. Cet article décrit certaines de ses manifestations telles qu'elles ont été rapportées dans les médias écrits, télévisés ainsi que sur Internet. L’analyse de messages de condoléances envoyés sur un site permet de mieux saisir les dimensions saillantes des représentations de cette mort et des réactions mondiales qu'elle a suscitées.
Ce document est protégé par la loi sur le droit d'auteur. L'utilisation des services d’Érudit (y compris la reproduction) est assujettie à sa politique d'utilisation que vous pouvez consulter en ligne.

https://apropos.erudit.org/fr/usagers/politique-dutilisation/ 


\section{Résumé}

L'expression du deuil public s'est manifestée de façon particulièrement éloquente lors de la mort de la princesse Diana en 1997. Cet article décrit certaines de ses manifestations telles qu'elles ont été rapportées dans les médias écrits, télévisés ainsi que sur Internet. L'analyse de messages de condoléances envoyés sur un site permet de mieux saisir les dimensions saillantes des représentations de cette mort et des réactions mondiales qu'elle a suscitées.

Mots clés : Lady Diana - funérailles deuil - réactions affectives - médias.

\section{Abstract}

The expression of public mourning after the death of Princess Diana in 1997 was particularly eloquent. This article describes some of the manifestations as they were reported in the written media, on television and on the Internet. The analysis of the messages of condolences sent to an Internet site provide a better understanding of the salient dimensions of the representations of this death and the international reactions it generated.

Key words: Lady Diana - funerals mourning - affective reactions - media.

\section{La mort de Lady Diana Un exemple de deuil public}

\author{
Joseph J. Lévy, Ph.D., \\ professeur, Département de sexologie, UQÀM. \\ Isabelle Toupin, M.A., \\ professionnelle de recherche, \\ Département de sexologie, UQÀM.
}

\section{Introduction}

Dans l'un des articles fondamentaux sur la compréhension des rites et des émotions rattachés à la mort, Hertz (1907), s'interrogeant sur les variations interculturelles et intraculturelles notées par les ethnographes quant à l'intensité des réactions et des sentiments face au décès d'une personne, avançait que ceux-ci semblent dépendre du statut social du défunt. Ainsi, lorsque ce statut est très élevé, ces réactions sont très vives, alors que dans le cas inverse, elles peuvent être atténuées et, dans certains cas, absentes, par exemple lors du décès d'enfants en bas âge. Hertz conclut à partir de son analyse que la mort

ne se borne pas à mettre fin à l'existence corporelle visible d'un vivant, elle détruit du même coup l'être social greffé sur l'individualité physique auquel la conscience collective attribuait une importance, une dignité plus ou moins grandes. La société communique aux individus qui la composent son propre caractère de pérennité parce qu'elle se sent et se veut immortelle, elle ne peut croire normalement que ses membres, surtout ceux en qui elle s'incarne, avec qui elle s'identifie sont destinés à mourir (p. 69-70).

Cette perspective peut aider à comprendre l'intensité des réactions du public qui se manifestent lors de la mort de personnes dans lesquelles l'investissement social est considérable: figures politiques majeures, militaires, vedettes de cinéma, explorateurs de l'espace, saints et héros.

\section{LA MORT DES GRANDS}

Les Oraisons funèbres de Bossuet constituent l'un des exemples les plus connus de l'expression publique de la mort des Grands. Les panégyriques prononcés en leur honneur et qui reprenaient d'ailleurs une tradition déjà présente dans la Grèce et la Rome antiques s'inscrivaient dans un rituel de funérailles élaboré. Dans l'une de ses lettres, madame de Sévigné décrivait comment ce type d'événement était annoncé par des affichettes distribuées dans les maisons, tandis que de grandes affiches étaient collées sur les murs pour inviter le public à participer aux cérémonies funéraires au cours desquelles ces discours étaient prononcés. Déjà, à cette époque, une certaine forme de médiatisation de la mort des personnes illustres était présente, une tendance qui ne fera que s'accentuer au cours des siècles suivants. La multiplication des médias, tant écrits que visuels, et leur influence de plus en plus mondiale, contribueront à mettre en relief la dimension 
spectaculaire de ces cérémonies et leur impact sur le grand public. Leur analyse ethnographique et politique dans les sociétés contemporaines montre ainsi les soubassements religieux de ces pratiques, même si elles s'inscrivent dans un contexte laïque :

Les funérailles des Grands nous renseignent en général sur les formes symboliques des croyances collectives à une époque donnée; au delà de leur objet propre, elles constituent de grands rassemblements communautaires où le corps du mort tient lieu d'eucharistie entre les vivants (Juilliard, 1999, p. 33).

Cependant, ces études tendent surtout à considérer la dimension des rituels funéraires sans tenir compte, de façon précise, des réactions affectives du public et de leurs significations. La mort de Lady Diana, la princesse de Galles décédée dans un accident de voiture à Paris à la fin d'août 1997, permet à cet égard de mettre en relief, de façon exemplaire, les processus associés aux rituels mortuaires modernes et à l'expression du deuil. Celui-ci dépasse le cadre familial, intime, pour s'inscrire dans des formes d'expression publiques qui se situent à différents niveaux: celles des personnalités politiques, des médias et du grand public. En ce sens, comme le note Ang (1997), la mort de cette princesse constitue l'un des événements culturels populaires les plus importants du $\mathrm{XX}^{\mathrm{e}}$ siècle et nous tenterons ici de cerner quelques-unes de ses configurations les plus saillantes.

\section{LA MORT DE LADY DIANA : RÉACTIONS DES MILIEUX POLITIQUES}

La mort de Lady Diana a donné lieu, comme il se doit, à des commentaires de la part des représentants des États. Ainsi la famille royale étant directement affectée par cet événement, la reine d'Angleterre dans sa déclaration à la nation mettait en relief la complexité des émotions ressenties et la dimension collective du chagrin provoqué par cette perte:

Il n'est pas facile d'exprimer un sentiment de perte puisque le choc initial est souvent suivi d'un mélange d'autres sentiments : incrédulité, incompréhension, colère et préoccupations pour ceux qui restent [...] J'espère que demain nous pourrons tous, peu importe où nous sommes, nous joindre pour exprimer notre chagrin suite à la disparition de Diana [...] C'est une chance qui nous est donnée de montrer au monde entier une nation britannique unie dans le chagrin et le respect.
Ce lexique démontre une certaine retenue affective si on la compare à la déclaration publique de Tony Blair qui insiste au contraire sur la force des sentiments provoqués par cette mort où le deuil et le chagrin atteignent les fibres les plus profondes de la nation: "Nous sommes aujourd'hui une nation en état de choc, en deuil, plongée dans un chagrin qui nous fait souffrir très profondément. » Cette amplitude affective s'estompe chez les autres dirigeants nationaux qui feront surtout mention des affects liés à la tristesse. C'est le cas de Jean Chrétien ou de Bill Clinton qui déclarait: «Laissez-moi encore vous dire combien Hilary et moi sommes tristes suite au terrible accident qui a ravi la vie de la princesse Diana et celle de ceux qui l'accompagnaient. »

Lionel Jospin, alors premier ministre de France, se situera dans le même registre: «Ce que j'ai ressenti en apprenant la mort de Lady Diana, c'est qu'il était profondément triste que cette belle jeune femme, aimée par les gens et dont tous les faits et les gestes étaient scrutés, ait terminé sa vie si tragiquement en France, à Paris. » Les autres politiciens européens (Jacques Chirac, Boris Yeltsin et Roman Herzog), en présentant leurs sympathies, mettent surtout en relief la contribution au travail humanitaire que la princesse effectuait et l'affection que pouvaient lui porter leurs peuples, sans exprimer d'émotions particulières. Ces réactions semblent donc être plus détachées des référents affectifs pour se situer dans un contexte plus protocolaire, ce qui peut s'expliquer par un investissement affectif moins profond lié aux éthos nationaux.

\section{LA MORT DE LADY DI : RÉACTIONS DES MÉDIAS ÉCRITS}

Les journalistes des médias écrits mettent aussi en exergue la dimension affective liée à la nouvelle de la mort, revenant sur les détails de l'accident et la biographie de la princesse. À titre d'exemple, des articles parus dans la Presse Canadienne (http://www.canoe.com/Princess Diana/, 1997) reprennent dans les titres principaux les émotions intenses qui accompagnent le deuil : tristesse, perte, chagrin.

Le corps des articles rapporte les réactions des instances politiques et des citoyens à cet événement. Alors que les déclarations des premières mettent surtout l'accent sur son statut, sa personnalité et son travail communautaire, celles des citoyens interrogés semblent être plus axées sur la dimension affective, mettant en relief un lien de proximité quasi familial avec la princesse, dont les péripéties biographiques tant intimes que sociales semblent bien connues et suivies avec attention, d'où l'intensité ressentie de la perte. Cette proximité est prolongée par un sentiment de sollicitude à l'égard des jeunes princes privés de leur mère: "C'est une mère et maintenant elle est morte. C'est terrible. Je me sens si triste pour ses deux jeunes enfants » (31 août 1997). D'autres commentaires portent sur l'humanité de la princesse et le coup du sort qui s'acharne sur elle à un moment où sa vie semblait s'orienter vers un dénouement plus heureux: "Après tout, elle venait de tomber amoureuse et tout cela a fini si soudainement [...] Elle était justement en train d'essayer de vivre sa vie et de ne blesser personne» (31 août 1997). La couverture médiatique des funérailles (voir par exemple les articles À l'intérieur de l'abbaye: des funérailles pour la princesse, Associated Press, 6 septembre 1997 ; Les endeuillés autour du monde suivent les funérailles familiales à la télévision, 6 septembre 1997) décrit les réactions de deuil qui ont accompagné le service funèbre dans l'abbaye de Westminster qui a été suivi, selon les estimations, par 2,5 milliards de personnes à travers le monde, fascinées par la princesse Diana dont la mystique est associée à son statut, à la reconnaissance de ses actions altruistes et à son destin tragique.

Dans des articles moins factuels, d'autres journalistes se sont interrogés sur la portée sociale et religieuse de ces manifestations de deuil, adoptant des positions contradictoires. S'interrogeant ainsi sur la signification religieuse de cette mort, un article paru dans le journal en ligne Faith in Focus de l'Église réformée de Nouvelle-Zélande met d'abord en évidence la capacité des individus à exprimer leur chagrin et leurs sympathies lors d'un décès significatif. Loin d'être devenue indifférente au malheur, l'humanité fait la preuve qu'elle est capable d'un sursaut d'émotions qui démontrent l'existence d'une solidarité et d'un partage. Les réactions à la mort de Lady Diana seraient aussi liées à une identification poussée car «elle représentait les aspirations et les espoirs de beaucoup de gens. Ils voyaient en elle la jeunesse, la vivacité et la beauté, et dans une grande mesure, sa vie les aidait à trouver un sens à leur propre existence».

Cependant, à ces aspects positifs viennent s'ajouter des connotations plus problématiques dans la mesure où ces conduites s'éloignent des référents religieux chrétiens. Ainsi, elles sont associées à une forme de déification proche de l'idolâtrie et à une forme de canonisation publique visant à lui accorder un statut proche de la sainteté à la suite de la reconnaissance de ses actions charitables, une position que réfute l'Église réformée pour qui ces œuvres ne peuvent être sanctifiées puisqu'elles ne s'inscrivent pas dans un projet associé au Christ, d'où le repositionnement de son statut et la tentative de le ramener à une évaluation 
plus modeste : «Diana était simplement un autre être humain marqué par le péché, comme nous tous. Elle n'était pas plus spéciale que le reste de l'humanité et avait autant besoin du Christ que nous » (p. 3).

Cette perspective critique est reprise par ailleurs par plusieurs éditorialistes laïques (cités dans Rockwell, 1997) pour qui le chagrin intense et massif ressenti lors de cette disparition est le reflet d'une configuration socioculturelle problématique où les médias jouent un rôle central. Ils contribuent en effet à monter en épingle la vie des célébrités et à encourager leur culte, symbole de la vacuité de la civilisation postmoderne où la mort devient source d'excitation extrême. Pour Rockwell, par contre, rejoignant dans son analyse les hypothèses de Hertz, ces réactions sont l'expression du refus d'un égalitarisme moral qui place toutes les morts au même plan. La mort de Lady Diana est le signe d'un regain d'intérêt pour la monarchie qui représente le cœur de la nation et celui de la reconnaissance de l'importance des valeurs d'altruisme qu'elle incarnait. Éloignée de la politique dans un monde hyperpolitisé, son histoire de vie met en évidence des thèmes universels qui rejoignent la narrativité des contes de fées d'où l'affection intense, mal comprise des autorités, que lui portait un vaste public. Rockwell suggère par ailleurs que la liturgie entourant son enterrement pourrait contribuer à la restauration d'une solennité religieuse absente de l'ethos religieux contemporain. Pour Rockwell (p. 7), en résumé

\section{La gloire du phénomène Diana} provient de sa nature non politique. Mais, néanmoins, il illustre un aspect qui est profondément pertinent pour notre avenir politique : l'impuissance du politique quand il est confronté à un épanchement massif d'une passion publique. [...] Le phénomène Diana démontre que le sentiment des personnes ordinaires - leurs émotions, leurs priorités et leurs amours, leur attachement aux normes traditionnelles de beauté et de foi et leur intérêt pour ce drame humain [...] et cette mort tragique - est plus puissant que tout intérêt particulier [... ] Contrairement à la position des conservateurs officiels, et celle de tous les autres qui ont dénoncé la tristesse exprimée par des personnes en chair et en os, le phénomène Diana n'est ni une imposture ni une fabrication.

Les réactions à la mort de Lady Diana ont aussi donné lieu à des réflexions qui s'inscrivent dans une perspective féministe. Coleman (1997), constatant une différence de genre dans l'expression des sentiments,

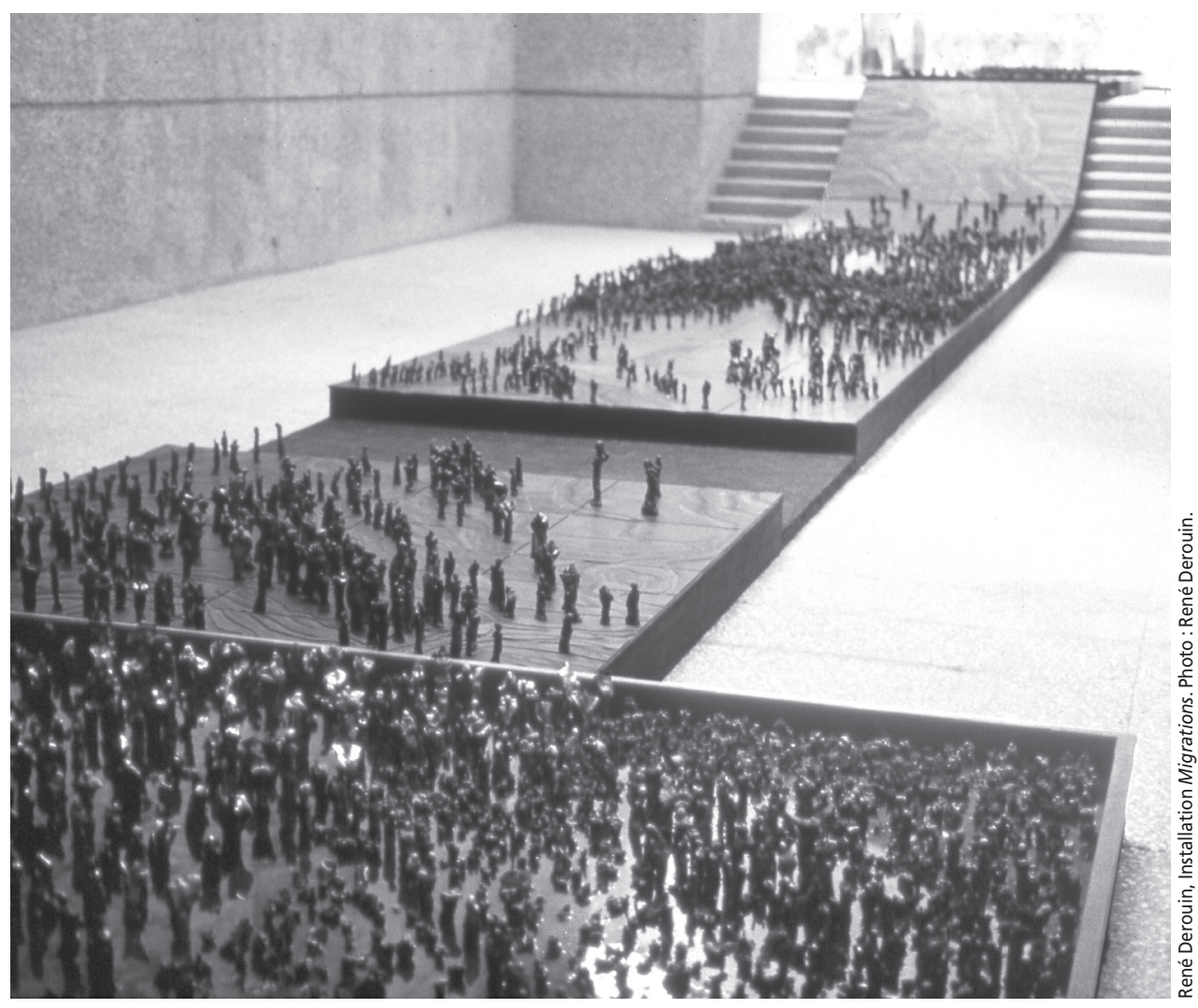

les hommes réagissant de façon atténuée à ce décès comparativement aux femmes enclines à une émotivité intense, attribue ces contrastes à des raisons multiples. Interviendrait ainsi la prégnance des modèles inspirés des contes de fées qui continuent de servir à la socialisation des femmes: la féminité affirmée de la princesse, son rôle de mère, ses troubles alimentaires et ses problèmes familiaux, autant de préoccupations qui rejoignent nombre de femmes. Ses capacités d'affirmation de soi et son refus de se conformer aux diktats sociaux pourraient avoir contribué à cette identification profonde par-delà les différences de classe ou de nationalité, des enjeux auxquels les hommes seraient moins sensibles, considérant la princesse plutôt comme un symbole sexuel. De plus, son projet de vie qui s'orientait vers une autoréalisation personnelle axée sur des objectifs altruistes rejoignait des aspirations féminines et donc un transfert affectif soudainement interrompu par sa mort.

\section{LA MORT DE LADY DIANA : RÉACTIONS DES MÉDIAS TÉLÉVISÉS}

Les canaux de télévision, qui ont souvent manipulé les événements entourant la mort des Grands de ce monde pour mettre sur le marché un produit communicationnel à même de river le public à leurs programmes (Dayan et Katz, 1996 ; Stark, 1997 ; Juillard, 1999), se sont attachés aux côtés sensationnels des conditions de l'accident, à une biographie détaillant les vulnérabilités personnelles, familiales et amoureuses de la princesse. Ils ont aussi insisté sur les réactions du public dont les larmes ou les rituels spontanés ont été mis en exergue (dépôt de bouquets de fleurs, de messages, de peluches, allumage de bougies au palais de Kensington, résidence de la défunte et autres endroits dans le monde), contribuant ainsi à propager un mimétisme affectif. La cérémonie funéraire, un moment crucial dans le processus de deuil, a donné lieu à une mise en scène dramatique et sa retransmission a contribué à la construction d'un ethos commun entre les spectateurs, permettant selon Stark (1997, p. 2,3) «à la nation et maintenant au monde d'être témoin d'un rituel direct et de partager son chagrin collectivement», mais simultanément et paradoxalement d'amener «l'endeuillé à exprimer son chagrin de façon privée, dans le confort de sa maison [...]. Le petit écran procure au spectateur une forme de deuil virtuel », mais en même temps il met en scène la réalité de la mort, un événement tabou et occulté. Ce deuil collectif, comme l'a montré Pina (1999) dans une comparaison entre les funérailles de Lady Di et celles de Mère Teresa dont les décès sont survenus presque au même moment s'inscrit dans une recherche «de la communion avec la douleur des autres » comme une tentative

de partager, autour d'un même corps, une même prière et un même sentiment. Il y a une norme sociale qui 
s'élabore alors autour de ce «j'ai tout vu, j' y étais » qui n'a d'autre signification que le plaisir de rentrer dans la masse, de se voir identique à d'autres. Se savoir relié au monde par l'intermédiaire d'un seul écran, se vouloir proche des autres tout en gardant ses distances, voilà bien l'arsenal que nous propose la télévision et aujourd'hui, Internet (p. 88).

\section{LA MORT DE LADY DIANA : RÉACTIONS SUR INTERNET}

Ce médium tout comme la télévision est, selon les critères définis par McLuhan (1964), un médium « froid ». Il demande en effet une forte implication des participants pour compléter les trous dans l'information transmise, contrairement aux médias chauds (où l'information présentée est plus complète et ne nécessite donc pas un investissement important de l'audience, comme c'est le cas pour les médias écrits). Internet implique en effet un haut degré d'investissement de la part de l'utilisateur qui participe activement à la recherche et à la création d'informations à travers un ensemble d'outils de communication (accès aux sites, courriels, salons de clavardage, messagerie instantanée, babillards, groupes de discussion) associés à l'interactivité, l'hypertextualité et la connectivité qui ont contribué à son succès. Ainsi, Internet permet une implication active avec la création de sites personnels ou collectifs, la participation à des salons de clavardage ou des groupes de discussion qui obéissent à des dynamiques locales et transnationales. La créativité inhérente à la Toile s'est exprimée de façon particulièrement évidente lors de la mort de Lady Diana, avec la mise en réseau de milliers d'internautes venus chercher de l'information et exprimer leur émotion sur des sites sur lesquels pouvaient être discutés et transférés des commentaires, des articles, des prises de position et des messages de condoléances provenant des quatre coins du monde. Giussani (1997) a ainsi montré comment, dès l'annonce du décès de Lady Diana, plusieurs sites de commémoration - dont la plupart sont aujourd'hui disparus - se sont rapidement mis en place pour permettre au public de partager ses réactions. Le site officiel de la monarchie britannique (British Monarchy : http://www.royal.gov.uk/) s'est ainsi doté d'une page de condoléances, alors que les groupes de discussion déjà existants, comme Alt. talk.royaly, newsgroup, s'emparaient aussi de cet événement. Ces messages constituent un matériau de recherche significatif pour aider à cerner les représentations de la mort et l'expression des émotions rattachées à des événements importants.

\section{LA MORT DE LADY DI : ANALYSE D'UN SITE DE CONDOLÉANCES}

Nous illustrerons ici, à travers l'analyse d'un échantillon non représentatif de messages recueillis sur le site Britannia.com/ diana/message-11.html qui rassemble plus de 1000 messages, ces représentations. L'ensemble des messages rédigés en anglais a fait l'objet d'une analyse lexicale à partir de laquelle les mots les plus saillants ont été repérés puis contextualisés. Complémentairement, près de 250 messages ont été codifiés afin de mieux cerner les dimensions marquantes et dont nous présenterons quelques exemples.

Ces messages signés, de courts textes d'une longueur moyenne de sept mots, proviennent de plusieurs pays, en particulier des États-Unis. Ils sont signés par des individus, des couples ou des familles et adressés directement aux enfants de Lady Di, les princes William et Harry, à la princesse Diana, comme si elle était encore vivante, ou ils n'ont pas de destinataire spécifique. Le contenu de ces messages met en relief la réception de l'événement, les émotions associées au décès, le devoir de mémoire, les représentations religieuses, les représentations de la défunte, ses rôles, ses qualités et sa contribution au monde.

Les réactions de stupéfaction à la mort de la princesse rejoignent la première phase de deuil, celle du choc à l'annonce du décès. Celle-ci est ainsi connotée le plus souvent comme un événement tragique, choquant, soudain et terrible qui fait irruption dans l'existence des répondants par l'intermédiaire d'une nouvelle entendue à la télévision, lue dans les journaux ou transmise par la rumeur. La dimension universelle de cet événement est aussi soulignée, quelquefois accompagnée d'un sentiment d'incrédulité et de sidération. Inquiets des répercussions de ce choc sur les enfants princiers, plusieurs se préoccupent de leur capacité à absorber cette situation nouvelle, d'où leur sollicitude à leur égard. Ce décès, pour un très grand nombre de rédacteurs, est considéré comme une perte majeure que connotent les adjectifs utilisés (vraie, immense, irréparable) qu'ils vivent en communion avec les enfants de la princesse. Dans le registre des sentiments, le plus fréquent renvoie à celui de vide et de manque intenses. Ce sentiment de proximité, dans des cas plus rares, peut être amplifié en associant cette mort à celle d'un membre de la famille immédiate. Ce n'est pas seulement la personne qui est regrettée mais certains de ses attributs physiques ( "Votre beau visage »; " son sourire et ce regard charmant») et moraux ("Votre personnalité aimable et aimante», "votre amour et votre force ») qui sont rappelés.
Cette perte est associée à plusieurs sentiments : la peine à la fois personnelle et collective, le chagrin et la douleur que les références aux larmes confirment. Par contre, les références liées directement au deuil sont moins fréquentes. Ces messages utilisent aussi souvent les expressions courantes de condoléances ou de sympathies et font fréquemment référence au devoir de mémoire en insistant sur le retentissement à long terme de cet événement qui se voit inscrit au plus profond de la mémoire de l'individu symbolisée par le cœur. La métaphore du cœur semble renvoyer au processus d'intériorisation qui permet d'assurer la présence intérieure du disparu. Comme l'a noté Dolto (s.d.) : « Il semble que c'est au cœur que soit réservée la projection du lieu focal où l'être humain situe symboliquement ses sentiments d'identification, de confiance, de sécurité passive ou active et d'échanges affectifs avec son semblable humain.» Cette métaphore est d'ailleurs utilisée de façon répétée pour désigner la princesse ("Princesse des cœurs») et son influence sur le public, ou pour exprimer les sentiments de partage et souligner le souvenir impérissable qu'elle laissera.

Ces promesses de mémorialisation sont renforcées par l'usage fréquent des adverbes comme «toujours », «jamais », " pour toujours » qui soulignent qu'elle est inoubliable à cause de sa personnalité ou du retentissement de sa bonté. Les sources de consolation sont pour plusieurs à chercher dans le recours à la foi et la religion. Ainsi, nombreuses sont les références à Dieu, alors que les allusions à Jésus-Christ sont pratiquement absentes du corpus. Les rédacteurs sont ainsi enclins à insister sur une forme rhétorique comme celle «Que Dieu bénisse » ou des formulations semblables. L'aide divine est aussi appelée pour assurer le repos de l'âme de la princesse qui continue de vivre dans un au-delà associé souvent à un paradis où elle est unie à Dieu.

Les constructions religieuses incluent aussi des croyances aux anges qui accompagnent la princesse dans un au-delà éternel ou qui désignent sa transfiguration en un être éthéré : «Que les anges transportent son âme au paradis »; "Le paradis comprend un autre ange magnifique » qui continue à veiller sur ses enfants et à les protéger. Ces messages sont aussi l'occasion d'idéaliser la princesse et de faire son éloge, de valoriser sa contribution familiale tout comme ses activités sociales et de la présenter comme un modèle à suivre en insistant sur ses qualités personnelles. Dans les représentations associées à ses rôles sociaux, la fonction maternelle est centrale et plus de $60 \%$ des messages en font mention, insistant sur ses aptitudes particulières dans ce domaine par 
sa mise en valeur comme mère « aimante », « fantastique », «belle», « attentionnée», «bien-aimée », "dévouée».

Présentée comme un modèle de rôle à suivre, un guide admiré pour son travail humanitaire auprès des enfants, des pauvres et des malades, pour sa compassion et son altruisme, les surnoms qui lui sont attribués - "Princesse des cœurs », "Princesse du peuple», "Reine de cœur» et «Robin des Bois moderne»- mettent en relief sa proximité avec les démunis du monde. Source d'inspiration pour ses enfants, pour l'ensemble des femmes et l'humanité tout entière, comparée à une étoile brillante, son héritage continuera dans le futur à inspirer d'autres vocations. En particulier, ses enfants sont appelés à suivre ses traces, à reprendre son flambeau et à prolonger son œuvre, sa mort servant d'exemple d'amour oblatif et d'abnégation. Ce processus de deuil s'ouvre donc chez plusieurs sur une identification à la personne décédée et sur une forme d'imitation de ses conduites, un mécanisme qui sert à surmonter la perte et à lui donner un sens positif. Ce faisant, la mort devient plus acceptable et le deuil dépassé pour s'ouvrir sur l'assurance d'un prolongement dans l'éternité, puisque son souvenir et son exemple continueront de briller, comme "une étoile [...] qui brille au dessus de nos têtes».

\section{CONCLUSIONS}

Dans le contexte social contemporain, la mort est généralement un sujet socialement abordé avec réticences. Pourtant, le décès de personnes institutionnellement significatives, comme le sont les figures publiques, ici la princesse Diana, met en relief les mécanismes collectifs de deuil par lesquels les individus réaffirment leurs liens sociaux que les médias modernes aident à mettre en scène et à dramatiser.

À travers des cérémonies funéraires publiques dans lesquelles les distances sociales se trouvent pour un moment abolies, la société retrouve une sorte d'unanimité de surface qu'expriment des sentiments intenses et partagés, chagrin, tristesse, peine, rattachés à la prise de la conscience de la perte d'un individu qui représente les aspirations sociales les plus élevées. La figure mythique de la princesse, dont la trajectoire personnelle et sociale fait d'elle une héroïne que sanctionne une mort inattendue, rejoint ainsi le panthéon des héros élevés à un statut particulier et dont l'influence subsiste même après leur trépas.

En ce sens, la mort corporelle n'abolit pas sa présence mais la transfigure, la projetant à un niveau de spiritualité extrême où elle rejoint le divin, un processus par lequel la victoire de la mort est remise en question, alors que les vivants, à travers les processus de mémoire, incorporent la personne décédée et réaffirment la valeur et l'exemplarité de sa vie dans un mouvement de partage qui passe par une expression physique et verbale.

À cet égard, Internet fournit un outil particulièrement bien adapté à cet objectif dans la mesure où il se prête à un usage personnalisé, qui s'articule à des réseaux de proportions mondiales. Ceux-ci amplifient les messages et les répercutent quasi instantanément. L'exemple du site anglais dont nous avons tiré les messages de condoléances analysés ici montre bien la portée de ce nouveau mode de communication dans la construction des représentations de la mort et le partage des émotions. Cette perspective n'est pas sans rejoindre certaines des analyses de Mauss (1921) qui, dans son article sur L'expression obligatoire des sentiments, montrait que bon nombre d'expressions orales, de sentiments et d'émotions dans les sociétés préindustrielles obéissent à des contraintes collectives :

Mais toutes ces expressions collectives, simultanées, à valeur morale et à force obligatoire des sentiments de l'individu et du groupe, ce sont plus que de simples manifestations [...] On fait donc plus que de manifester ses sentiments, on les manifeste aux autres, puisqu'il faut les manifester.

On se les manifeste à soi en les exprimant aux autres et pour le compte des autres (p. 7-8).

$\mathrm{Ne}$ retrouve-t-on pas, à travers des moyens modernes, des fonctions similaires dans cet engouement pour la rédaction de ce type de messages, dont il conviendrait d'étudier plus en détail les différentes facettes?

\section{Bibliographie}

ANG, Ien (sous la direction de) (1997). Planet Diana: Cultural Studies and Global Mourning. Kingswood, University of Western Sydney Press.

COLEMAN, Sarah (1997). Princess Diana's Death. A Feminist Response. Http://www. feminista. com/archives/v1no6/coleman.html. 4 pages. Consulté en février 2004.

DAYAN, Daniel et KATZ, Elihu (1996). La télévision cérémonielle, Paris, Presses universitaires de France.

DOLTO, Françoise (s.d.). Le cour, expression symbolique de la vie affective. Http://www. sospy.com/Bilbiopsy/Biblio10/biblio002.htm. Consulté en février 2004.

FAITH in FOCUS (1997). The Death of Lady Diana. Http://www.reformed-churches.org. nz/resources/fnf/a61.htm. Octobre. 3 pages. Consulté en février 2004.

GIUSSANI, Bruno (1997). Mourning Diana: Online Chat Becomes a Book of Condolences. Technology Cybertimes. The New York Times on the Web. @ Septembre. Http://www. nytimes.com/library/cyber/euro/090297euro. html. 2 pages. Consulté en février 2004.

HERTZ, Robert (1907, 1928).«Contribution à une étude sur la représentation collective de la mort ». Dans Sociologie et Folklore, Paris, Presses universitaires de France, p. 14-79. Version PDF. Http ://www.uqac.uquebec.ca/ zone30/classiques_des_sciences_sociales/ classiques/hertz-robert/socio_religieuse_ folklore/socio_religieuse_html

JUILLARD, Jacques (sous la direction de) (1999). La mort du roi. Essai d'ethnographie politique comparée, Paris, Gallimard.

MCLUHAN, Marshall (1964). Understanding Medias: The Extensions of Man, New York, McGraw-Hill.

MAUSS, Marcel (1921). "L'expression obligatoire des sentiments ", Journal de psychologie, 18. Version PDF. Http://www.uqac. uquebec.ca/zone30/Classiques_des_sciences_ sociales/classiques/mauss_marcel/essais_ de_socio/T3_exprsssion_sentiments/ expression_sentiments.html

PINA, Christine (1999). "Lady Di et Mère Teresa: deux saintes cathodiques? ", Religiologiques, $\mathrm{n}^{\circ} 19$, septembre, p. 79-95.

ROCKWELL, Llewellyn H. Jr (1997). St Diana. http://www.salon. com/sept97/diana970923.html. 8 pages. Consulté en février 2004.

STARK, Steven D. (1997). A Macabre Sporting Event. Why Funeral Play so Well on TV. http:// www.salon. com/sept97/media/media970911. html. 4 pages. 